Table des matières du tome LXXXII, fascicule 4

-.. Pages

H. Wang, Notes on a class of tiling problems . . . . . . . . 295-305

A. S. Troelstra, Non-extensional equality. . . . . . . . . . . 307-322

R. Fraïssé, Multirelation et àge 1-extensifs . . . . . . . . . . . . 323-330

U. Felgner, $s_{1}$-Kategorische Theorien nicht-kommutativer Ringe . . . . 331-346

W. Schwabhäuser and L. W. Szczerba, Relations on lines as primitive

notions for Euclidean geometry . . . . . . . . . . . . . 347-355

F. Galvin and J. Larson, Pinning countable ordinals. . . . . . . . . 357-361

L. Henkin and D. Resek, Relativization of cylindric algebras . . . . . 363-383

M. Makkai, Errata to the paper "Genoralizing Vaught sentences from $\omega$ to strong cofinality $\omega "$ ", Fundamenta Mathematicae 82 (1974), pp. 105-119

\title{
Notes on a class of tiling problems
}

by

\author{
Hao Wang (New York)
}

Les FUNDAMENTA MATHEMATICAT publient, en langues des congrès internationaux, des travaux consacrés à la Théorie des Ensembles, Topologie, Fondements de Mathématiques, Fronctions Réelles, Algèbre Abstraite

Ce volume paraît en 4 fascicules

Adresse do la Rédaction et do l'Échango: fundamenta MATHEMATiCAE, Sniadeckich 8, 00-950 Warszawa (Pologne)

Tous les volumes sont ì obtenir par l'intermédiaire do ARS POLONA-RUCH, Krakowskie Przedmieścio 7, 00-068 Warszawa (Pologne)

Corespondence concerning editorial work and mimuseripts should be addressed to: FUNDAMENTA MATHEMATICAE, Sniadeekich 8, 00-950 Warszawa (Poland)

Correspondence concerning exchange should be addressed to:

INSTITUTE OF MATHEMATICS, POLISH ACADEMY OF SCIENCES, Exchange, Sniadeckich 8, 00-950 Warszawa (Poland)

The Fundamenta Mathematicae are available at your bookseller or at ARS POLONA-RUCH, Krakowskie Przedmieścio 7, 00-068 Warszawa (Poland)

\begin{tabular}{l} 
DRUKARNIA UNIWERSYTETU JAGIELLONSKIEGO W WRAKOWIE \\
\hline
\end{tabular}
Abstract. The class of problems considered here was at first 'called the "domino problems" and has found extensive applications with regard to the decision problem of the predicate calculus. This paper includes, apart from a brief survey of work related to this class of problems, a number of isolated results which have been obtained over the years. These results mostly have little direct connection with mathematical logic but may be, for that very reason, of some interest to a wider circle of mathematicians.

The class of tiling problems deals with the following general situation. Suppose that we are given a finite set of unit squares with colored edges, placed with their edges horizontal and vertical. We are interested in tiling the plane with copies of these tiles obtained by translatives only. The tiles are to be placed with their vertices at lattice points, and abutting edges must have the same color. The first question, the unrestricted tiling problem, is whether there is a general method of deciding which finite sets of coloried squares are solvable (i.e., can be used to tile the plane in this way). The second question (closely related to the first, see below) is whether every solvable set has a periodic solution (i.e. yields a square of some size which repeats to cover the plane). If we think of the first quadrant instead of the whole plane, it is more convenient to speak also of the origin-constrained (i.e. the tile at the origin is restricted to a given subset) and the diagonal-constrained (i.e. the tiles along the main diagonal are restricted) tiling problems. These apparently frivolous problems have led to various interesting investigations. And it is my purpose here to give a number of fragmentary results mostly obtained in discussions with colleagues and students some time ago. In particular, several of the basic ideas are due to Edward F. Moore. I shall begin with a brief historical survey of some of the results in the literature.

Around the beginning of 1960, while continuing my work on the mechanization of mathematical arguments, I was diverted into a study of the theoretical problem of deciding the class of sentences with the simple quantifier prefix AEA in elementary logic. After a period of effort, I succeeded in transforming the decision problem into the easily understandable combinatorial or geometrical tiling problem (called the "do1 - Fundamenta Mathematicae, T. LXXXXI 
mino problem" by a colleague). This greatly facilitated not only my communication with my colleagues at the industrial laboratory who were mostly ignorant of mathematical logic, but also the ability to focus attention on the mathematical core of the original decision problem. At this time, I also discovered that the origin-constrained tiling problem is unsolvable, because operations of any Turing machine can be simulated by a particular tiling problem. The formulation of the tiling problems was written up in May 1960 and published in January 1961 ([17]), and the result on the origin-constrained problem was written up in August 1961 for circulation (see [18]).

In the autumn of 1961, I lectured on these things. And in collaboration with Kahr and Moore, I was able to show that the diagonal-constrained tiling problem was also unsolvable for much the same reason as the originconstrained problem. We were able to infer that the decision problem for the AEA case is unsolvable. In fact, the AEA sentences with only dyadic predicates form a reduction class. These results were published in [11]. Shortly afterwards, Kahr further refined the result to eliminate all but one dyadic predicate, using only monadic predicates otherwise. A summary of this last result is included in [10] and [19], and the full proof is given in Kahr's MIT Ph. D. dissertation (June 1962). Afterwards Berger, another student of mine, demonstrated that the (original) unrestricted tiling problem is also unsolvable (in his Harvard dissertation, June 1964; a briefer version appeared as [1]).

Over the years, there have appeared a number of papers related to these tiling problems and decision problems. The following items have come to my attention. There are three pairs of natural subclasses of the AEA sentences with dyadic predicates only. Two of these three pairs have been shown to be decidable (see [2] and also [19]). The remaining pair is, surprisingly, shown to be undecidable by S. Aanderaa (in his Harvard dissertation, August 1966). Elaborations, extensions, and simplifications of [10], [11], and [19] are contained in the papers by Genenz, Hermes, and Maslov ([3], [4], [8], [9], and [12]). On the question of nonperiodicity, Berger's published proof of the unsolvability of the tiling problem contains a complex solvable set of tiles with no periodic solution. In his dissertation, he includes a simpler set with 104 tiles. In April 1966, H. Lauchli sent me a nonperiodic set with 40 tiles which, as far as I know, has not been published. In [15], Robinson has gone into the solvability and the periodicity problems carefully and obtained more economical solutions. In a somewhat different direction, Hanf has shown in [6] that, under the origin constraints, there is a finite solvable set of tiles which has no recursive solution. This was extended by Myers (in [13]) to the unrestricted case. The tiling obtained by Hanf can be described by a 1 -trial predicate (a concept of Putnam [14]). Carl
Jockusch has found a solvable finite set of tiles which has no $m$-trial tiling for any $m$. Hanf's work was aimed at proving Conjecture I of [7], but these results have failed to settle his conjecture.

Let $N$ be the class of unsolvable tile sets, $F$ be the class of tile sets with periodic solutions, and $J$ be the class of solvable sets without periodic solutions. It is proved in [5] that the classes $N, F, J$ are pairwise recursively inseparable.

I proceed to list a number of fragmentary results, often omitting complex constructions.

With the origin constraint, it is possible to force special solutions with amusing properties. One example is to distinguish prime from composite numbers, first done by Edward F. Moore and then simplified by M. Fieldhouse.

1. There is a set of 30 tiles, including three tiles $A, P$ and $C$, such that if $A$ is required to appear at the origin, the set has a unique solution in which $P$ and $O$ occur respectively at the prime and composite positions in the first row.

A variant formulation of the tiling problem is to color the corners rather than the edges so that each tile can naturally be represented by a 2 by 2 matrix giving the colors of the four corners. It is easy to show that this formulation is equivalent to the other one in an obrious sense.

It can be verified that permitting all rotations or all reflections would make all sets of tiles solvable. If, however, we use regular hexagons instead of squares and allow reflections and rotations, we have:

2. Given a set of square plates, we can find effectively a set of regular hexagons such that there is a one-to-one correspondence between the solutions of the two sets. Conversely, given a set of regular hexagons, we can also find effectively a corresponding set of squares.

Given a set of squares $A_{1}, \ldots, A_{n}$, we introduce a set of $2 n+1$ hexagons with $6+n$ new colors as follows. A "cementing" hexagon:

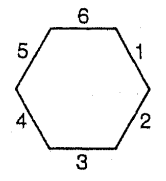

Suppose $A_{i}$ is:

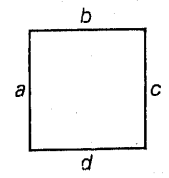


Introduce two hexagons with a new color $a_{i}$ :
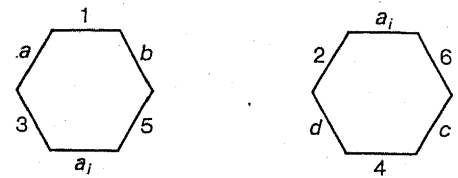

It can be seen that on account of the cementing piece, each pair of hexagons can only be used as a unit thus:

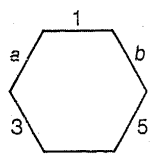

$a_{i}$

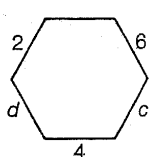

Conversely, given a set of regular hexagons $H_{1}, \ldots, H_{m}$, we give one block of three squares for each of the 12 positions for each $H_{i}$, using two new colors for each position. For example, if $H_{i}$ is

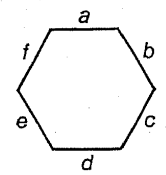

we use for the particular position the obvious combination of the three squares:
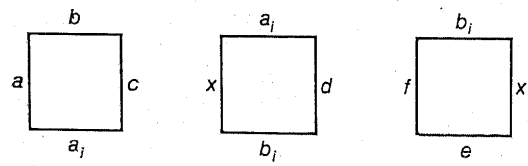

The letter $x$ indicates a new color used for every triple.

Hence, we get a set of $36 m$ squares with $24 m+1$ new colors.

We omit the detailed proofs of the two halves of Proposition 2.

It is convenient to speak of a "torus" when we have a square or rectangle of some size such that the top agrees with the bottom (in colors) and the left edge agrees with the right edge. As we have noted before, Berger and others have given solvable sets which yield no tori because they have no periodic solutions. An easier question had been considered and answered earlier, viz. to find a set which has a solution in which no torus occurs.

This uses an interesting construction due to Thue (see [16]), according to which the union $R$ of the infinite sequence obtained from $a$ by successively replacing $a$ by $a b, b$ by $b a$ contains no part $U y \nabla$ with $U y$ $=y \nabla$. Such a two-way infinite Thue sequence $R$ can be applied to design a pattern of $a$ 's and $b$ 's on the plane in which there is no "torus". We put the sequence $R$ horizontally on the plane and copy each symbol across the diagonal bisecting the first and the third quadrants. Then there can be no rectangle block which forms a torus. For, suppose there were such a block, say

$$
\begin{array}{llll}
x_{4} & x_{3} & x_{2} & x_{1} \\
x_{3} & x_{2} & x_{1} & y_{1} \\
x_{2} & x_{1} & y_{1} & y_{2}
\end{array}
$$

Then $x_{4} x_{3} x_{2} x_{1}=x_{2} x_{1} y_{1} y_{2}$ and, in particular, $x_{4} x_{3} x_{2}=x_{2} x_{1} y_{1}$. Therefore, $x_{4} x_{3} x_{2} x_{1} y_{1}$ would be a part $U x_{2} V$ such that $U x_{2}=x_{2} V$.

In order to find a set of tiles with a solution in which no torus occurs, we represent $a$ and $b$ by several tiles, taking into consideration the two neighbours in the same row. Since we do not permit $a a a$ or $b b b$, we need only six tiles:
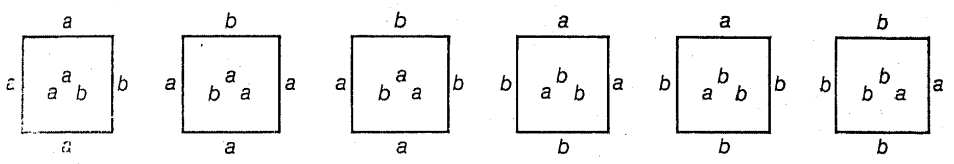

It can be verified that the pattern of $a^{\prime}$ s and $b$ 's described above can be simulated to any size. Hence, applying 5.1 to be proved below, we obtain:

3. There exists a set of six tiles which has a solution in which no torus occurs.

Incidentally, Thue also found that, for any alphabet with three or more letters, there are sequences in which no part is of the form $D D$. A sequence of this kind with three letters has been found which is simpler to describe than Thue's:

4. The sequence $T$ obtained from the sequence $R$ described above by substituting $c a$ for $a a, c b$ for $b b$ contains no part $D D$.

We recall that $R$ is the union of:

$R_{1}=a, R_{2}=a b, R_{3}=a b b a, R_{4}=a b b a b a a b, \ldots$

Consider now the even trims of $R$ beginning with $R_{4}: R_{4}, R_{6}$, etc. We observe that, because neither aaa nor $b b b$ occurs in $R$, the results 
of substituting $c a$ for $a a$ and $c b$ for $b b$ are composed of occurrences of only the following 4 -letter sequences:

$$
A=a c b a, \quad B=b c a b, \quad C_{1}=a c b c, \quad C_{2}=b c a c .
$$

We distinguish different occurrences of $c$ and use $c_{1}$ in a context bca, $c_{2}$ in a context $a c b$. Define $T_{1}, T_{2}, T_{3}$, etc. by:

$$
\begin{aligned}
T_{1}\left(a, b, c_{1}, c_{2}\right) & =a c_{2} b a b c_{1} a b, \\
T_{n+1}\left(a, b, c_{1}, c_{2}\right) & =T_{n}\left(A, B, C_{1}, C_{2}\right) .
\end{aligned}
$$

Then $T$ is the union of the $T_{n}$ 's and $T_{n}$ is obtained from $R_{2 n+2}$ by substituting $c a$ for $a a, c b$ for $b b$.

If $D D$ occurs in $T$, then there is some $T_{n}$ such that $D D$ occurs in $T_{n}$ and $T_{n}$ contains more letters following $D D$. Let $M$ be obtained from $D$ by substituting $A, B, C_{1}, C_{2}$ for $a, b, c_{1}, c_{2}$. Then $M M$ occurs in $T_{n+1}$, and $M$ begins with $a$ or $b$. Suppose $M=x N(x=a$ or $b)$. We then have $x N x N$ in $T_{n+1}$. The letter following $x N x N$ must also be $x$ because $x$ follows $N$ once in $T_{n+1}$ in $x N x N$. For example, if $x$ is $a$, then $M$ must begin with $A$ or $C_{1}$, but $C_{1}$ can only follow $B$, and $A$ can only follow $C_{1}$ or $B$. Hence, $M$ must end with $C_{1}$ or $B$. But $C_{1}$ can only be followed by $A$, and $B$ can only be followed by $A$ or $C_{1}$. Therefore, the part in $T_{n+1}$ after $x N x_{N} V$ must begin with $a$ (i.e., as head of $A$ or $C_{1}$ ). Hence, we have $x N x N x$ in $T_{n+1}$ and, therefore, $x K x K x$ in $R_{2 n+2}$, contradicting the fact that $R$ contains no part $U x V$ with $U x=x V$.

There are a number of more or less direct consequences of the infinity lemma concerned with solutions of sets of tiles.

5.1. For a given tile set $P$, if, for every $n$, there is a solution of size $n$ by $n$, then $P$ has a solution.

We consider partial solutions of size $2 n-1$ for $n=1,2, \ldots$ and make a tree such that a block $K$ of size $2 n-1$ by $2 n-1$ leads directly only to blocks of size $2 n+1$ by $2 n+1$ with $K$ in the center. The hypothesis and the fact that any part of a partial solution is also a partial solution ensures an infinite tree. Hence, the infinity lemma yields an infinite path in the tree which represents a solution of $P$.

5.2. A tile set is solvable over the whole plane if and only if it is solvable over a quadraint.

If it is solvable over the plane, we can of course get a solution over a quadrant by deleting the other quadrants from a given solution. Conversely, if it is solvable over a quadrant, then it has a solution of size $n$ by $n$ for every $n$. Hence, by 5.1, it has a solution over the whole plane.
5.3. Given a tile set $P$ for which it is possible to form two adjacent infinite rows such that for every $m$, any 1 by $m$ block occurring in the top row also occurs in the bottom row. Then the set $P$ is solvable.

Let $A$ be the given top row, $B$ be the given bottom row. For each $m$ and a 1 by $m$ block $C_{m}$ in $A$, there is a $D_{m}$ in $A$ such that $D_{m}$ can be cor rectly put on top of $C_{m}$, because $C_{m}$ also occurs in $B$. Repeating this process with $D_{m}$, and so on, we can obtain a partial solution of size $m$ by $m$. Hence, by 5.1, the set $P$ has a solution.

A solvable tile set is said to be minimal solvable if in every solution every tile of the set occurs.

5.4. Given a minimal solvable set, there exists an integer $n$ such that every tile of the set occurs in every $n$ by $n$ block in every solution.

Assume 5.4 false and we have for every $n$ some solution $S$ and some tile $T_{i}$ such that there is an $n$ by $n$ block not containing $T_{i}$. If the number of tiles is $k$, make $k$ trees $K_{1}, \ldots, K_{k}$ as in 5.1 so that $K_{i}$ includes all the $2 n-1$ by $2 n-1(n=1,2, \ldots)$ blocks which does not include $T_{i}$ in some solution. By our assumption, at least one of the trees, say $K_{i}$, must be infinite. This determines a solution in which $T_{i}$ does not occur at all, contrary to the hypothesis that the given set is minimal solvable.

5.5. If the (unrestricted) tiling problem is unsolvable, then there is a solvable set with no periodic solution.

This was observed in [17]. If every solvable set had periodic solutions, we would have the following situation. Either a set is solvable, there would then be some $n$ such that there is a torus of size $n$ by $n$. Or a set is unsolvable, then, by the infinity lemma, there would be some $n$ such that there is no solution of size $n$ by $n$. Hence, we would be able to test successively for each $n$, whether there is a solution or a torus of size $n$ by $n$. This process must terminate at some finite stage, and we would have a decision method for the tiling problem.

Of course, as mentioned before, we now know that the tiling problem is unsolvable and we possess also relatively simple examples of solvable sets with no periodic solutions.

5.6. Given a solvable tile set and an integer $n$, there exists a solution in which every occurring finite block of size no bigger than $n$ by $n$ occurs infinitely often.

Consider any given solution. Since there are only a finite number of tiles, there must be at least one which occurs infinitely often. Let $M I_{1}$ be the set of all tiles which occur infinitely often. Let $M_{2}$ be the set of all 2 by 2 blocks of tiles each of which occurs infinitely often in the solution and, in addition, consists only of members of $M_{1}, M_{2}$ is again not empty, since the set of 2 by 2 blocks which occur finitely often 
can oniy take up a finite area in the plane. In general, given $M_{n}$, the set $M_{n+1}$ of $n+1$ by $n+1$ blocks each of which occurs infinitely often and contains only members of $M_{1}, \ldots, M_{n}$ is not empty. Hence, by the infinity lemma, there is a solution with a member of $M_{1}$ in the center, a member of $M_{2}$ as the central 2 by 2 block, etc. This gives a solution in which every finite block occurs infinitely often in the original solution. To make sure that we get the desired solution for a given $n$, we repeat the process of eliminating blocks which occur only finitely often. Since there is only a finite number of distinct blocks of size no bigger than $n$ by $n$, this process must come to an end.

5.7. Every solvable set has a solution $S$ such that every finite block occurring in $S$ occurs infinitely often in $S$.

Given a solution $T$ and the set $K$ of all finite blocks occurring in $T$, consider the set $I$ of all subsets of $K$ such that a subset $A$ of $K$ belongs to $\mathcal{I}$ if there is a solution covered by $A$, i.e., in which all the occurring finite blocks (or, equivalently, just squares) belong to $A$. The set $L$ is not empty because $K$ belongs to it and it has minimal members. Take any minimal member $B$ and any solution $S$ covered by $B$. If there is any block in $B$ which appears only finitely often in $S$, we can eliminate it by 5.6, and $B$ would not be minimal. Hence, every block in $B$ must appear infinitely often in every solution $S$ covered by $B$.

5.8. If a solvable set $P$ has no periodic solutions, then it has as many distinct solutions as there are real numbers.

Since the set $P$ is solvable, it has, by 5.7 , a solution $S$ in which every occurring finite block occurs infinitely often. Hence, if an $n$ by $n$ block occurs in $S$, it must have two nonoverlapping occurrences. Begin with two occurrences in $S$ of a single tile $T$. There must be some $n_{1}$, such that the $n_{1}$ by $n_{1}$ blocks with $T$ at the center at the two places are different. Otherwise, the two infinite columns $C$ and $D$ containing the two occurrences of $T$ (or rows if they are in the same column) must be the same at corresponding positions, in which case we join the two occurrences of $T$ by a staircase and consider all analogous staircases between the two columns. Since there are infinitely many staircases, at least two must be identical. But then we can take the region $R$ bounded by $C, D$ and two identical staircases and repeat it up and down to get an infinite strip $S$ bounded by the modified columns $C^{\prime}$ and $D^{\prime}$. Since $C$ and $D$ are identical at corresponding places, the two vertical parts $V_{1}$ and $V_{2}$ bounding $R$ are identical. Therefore, we can also repeat the infinite strip $S$ and cover the plane. Since each column consists of repetitions of $V_{1}$ (or, what is the same thing, $\nabla_{2}$ ), there must be two infinite columns which are identical (i.e., without any staircase shift). Since there are infinitely many segments of rows bounded by the two columns, two of them must be identical. Therefore, we would have a torus, contradicting the hypothesis of nonperiodicity.

Hence, beginning with $T$ and its two occurrences, we can expand to two different $n_{1}$ by $n_{1}$ blocks. Each $n_{1}$ by $n_{1}$ block has two nonoverlapping occurrences which, for similar reasons, can be extended to two distinct bigger blocks. Hence, we have, by repeating the process, a full binary tree with as many infinite paths as there are real numbers. But each infinite path determines a solution.

Given an infinite set $K$ of solutions of a tile set $P$, a solution $S$ of $P$ is said to be a limit solution of $P$ and $K$ if every finite block in $S$ agrees with infinitely many solutions in $K$ over that block.

5.9. Every infinite set of solutions of a tile set has a limit solution; in other words, if there are infinitely many infinite paths, then there is a path on which every node appears in infinitely many infinite paths.

Suppose $K$ is an infinite set of solutions of the tile set $P$. Form a tree as follows: The nodes on the $n$th level of the tree consist of all $2 n-1$ by $2 n-1$ partial solutions of $P$ which coincide 'with infinitely many members of $K$ when these members are restricted to the $2 n-1$ by $2 n-1$ block centered at the origin. A node on the $n$th level is connected to a node on the $(n+1)$-st level if and only if the smaller partial solution comprises the center of the larger one. To see that the resulting tree is infinite, we need only verify that there is at least one node on every level. But this is true because there are infinitely many members of $K$, but (for fixed $n$ ) only finitely many blocks of size $2 n-1$ by $2 n-1$. Finally it is clear that only finitely many branches spring from each node. Hence, by the infinity lemma, the tree has an infinite path. This path describes a solution, which is a limit solution to the set $K$; i.e. every finite block of the solution coincides with the corresponding block in infinitely many members of $K$.

We mention incidentally an application of the infinity lemma in a different context.

5.10. If a Turing machine halts for every initial state and every initial tape (which may contain infinitely many marked squares), then there is a number $N$ such that the machine always halts before $N$ steps.

Consider at each moment $t$ the pair $\left(q_{t}^{i}, S_{t}^{i}\right)$, where $q_{t}^{i}$ is the state and $S_{t}^{i}$ is the symbol under scan at $t$. At the initial time $t=\mathbf{1}$, we have only a finite number of $\left(q_{1}^{i}, S_{1}^{i}\right)$. At each moment, from each $\left(q_{t}^{i}, S_{t}^{i}\right)$, we have only a finite number of $\left(q_{t+1}^{j}, S_{t+1}^{j}\right)$. Since the machine always stops, the tree contains no infinite path. But, by the infinity lemma, if there are altogether infinitely many finite paths, there is some infinite path. 
Hence there can be only a finite number of finite paths, and hence a finite bound $N$ to the height of all paths. The theorem is proved.

We conclude this paper by giving H. Lauchli's solvable set without periodic solution. The matching condition requires that the adjacent tiles have the same color (solid. or broken) and that lines must continue across edges. There are five classes of eight tiles each.
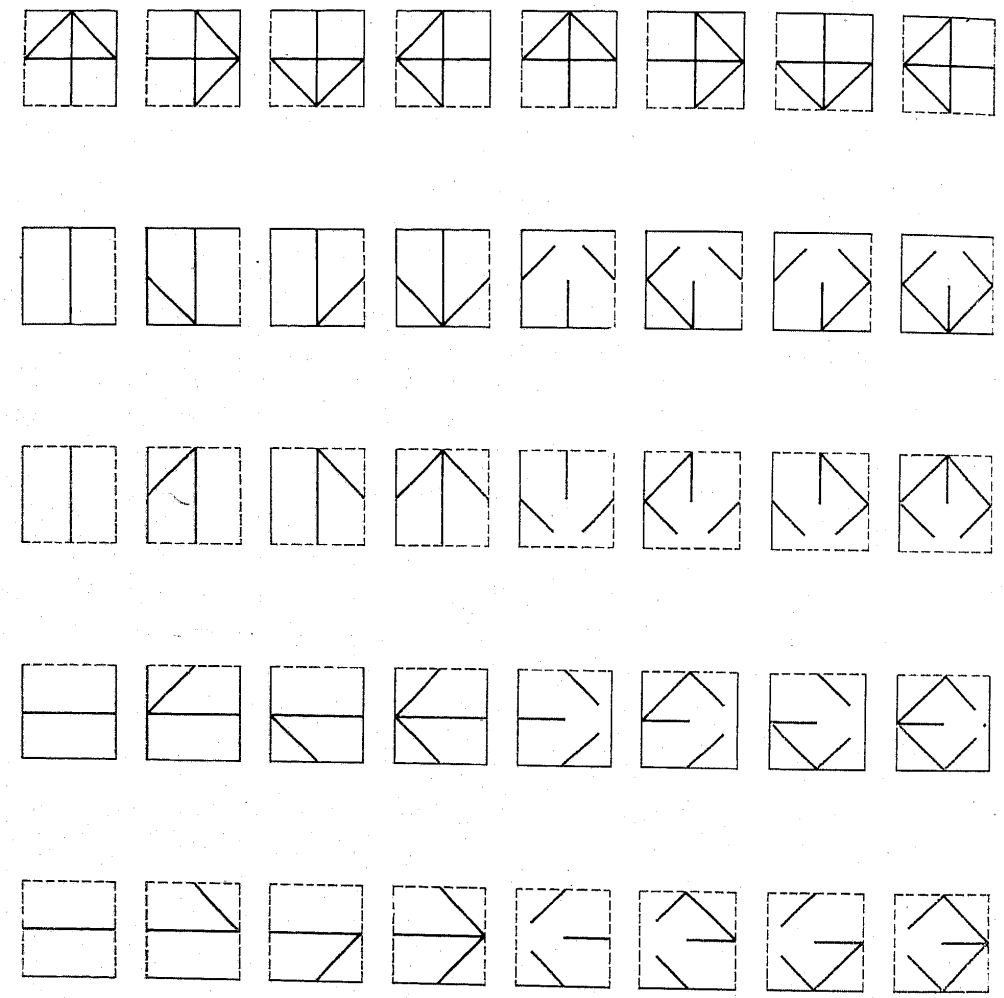

\section{References}

[1] R. Berger, The undeoidability of the domino problem, Mem. Amer. Math. Soc. 66 (1966), pp. 72

[2] B. Dreben, A. S. Kahr, and H. Wang, Olassification of AEA formulas by letter atoms, Bull. Amer. Math. Soc. 68 (1962), pp. 528-532.

[3] J. Genenz,
$*$ 1964, pp. 88
[4] J. Genenz, Untersuchungen zum Entscheidungsproblem, Münster 1965, pp. 44

[5] Gurjevits and Korjakov, Remarks on a paper of Berger on a problem of dominoes (in Russian), Sib. Math. Journal 13 (2) (1972), pp. 459-463.

[6] W. Hanf, Nonrecursive tilings of the plane I, submitted to J. Symb. Logic.

[7] - Model-theoretio methods in the study of elementary logic, Theory of Models 1965 pp. 132-145.

[8] H. Hermes, Entscheidungsproblem und Dominospiele, Selecta Mathematica II (1970), pp. 114-140.

[9] - A simpinfied proof for the unsolvability of the AEA case, Logic Colloquinm 1969 (1971), pp. 307-309.

[10] A. S. Kahr, Improved reductions of the Entscheidungsproblem to subclasses of AEA formulas, Mathematical Theory of Automata 1963, pp. 57-70.

[11] - E. F. Moore, and H. Wang, Entscheidungsproblem reduced to the AEA case, Natl. Acad. Sci. US 48 (1962), pp. 365-377.

[12] S. Ju. Maslov, The inverse method for logical calculi, Trudy Mat. Inst. Steklov. 98 (1968), pp. 26-87 (see § 12.3).

[13] D. Myers, Nonrecursive tilings of the plane II, to appear.

[14] H. Putnam, Trial and error predicates, J. Symb. Logic 30 (1965), pp. 49-57.

[15] R. M. Robinson, Undecidability and nonperiodicity for tilings of the plane, Invent. Math. 12 (1971), pp. 177-209.

[16] A. Thue, Über die gegenseitige Lage gleicher Teile gewisser Zeiehenreihen, Kristiania, 1913, pp. 67.

[17] H. Wang, Proving theorems by pattern recognition II, Bell Systems Technical J. 40 (1961), pp. 1-41.

[18] - An unsolvable problem on dominoes, Harvard Computation Laboratory report no. BL-30 (II-15), duplicated August 1961, bound Jan., 1962.

[19] - Dominoes and the AEA case of the decision problemi, Mathematical Theory of Automata 1963, pp. 23-56.

Reçu par la Rédaction le 30. 1. 1973 